\title{
Weyl sequences: Asymptotic distributions of the partition lengths
}

by

Anatoly Zhigluavsky (Cardiff) and Iskander Aliev (Warszawa)

\section{Introduction: Statement of the problem and formulation of the main results}

1.1. Weyl sequences. Let $\theta$ be an irrational number in $[0,1)$ and $x_{k}=$ $k \theta(\bmod 1)$ for $k=1,2, \ldots$ The collection of points $W_{n}(\theta)=\left\{x_{1}, \ldots, x_{n}\right\}$ is sometimes called the Weyl sequence of order $n$.

In the present work we derive asymptotic distributions of different characteristics associated with the interval lengths of the partitions of $[0,1)$ generated by $W_{n}(\theta)$. The main result establishes the two-dimensional asymptotic distribution of

$$
\left(n \min \left\{x_{1}, \ldots, x_{n}\right\}, n\left(1-\max \left\{x_{1}, \ldots, x_{n}\right\}\right)\right)
$$

as $n \rightarrow \infty$. It then yields a number of results concerning the asymptotic distributions of one-dimensional characteristics.

Assume that $y_{0, n}=0, y_{n+1, n}=1$ and let $y_{k, n}(k=1, \ldots, n)$ be the members of $W_{n}(\theta)$ arranged in increasing order. Define

$$
\text { (1) } \quad \delta_{n}(\theta)=y_{1, n}=\min _{k=1, \ldots, n} x_{k}, \quad \Delta_{n}(\theta)=1-y_{n, n}=1-\max _{k=1, \ldots, n} x_{k}
$$

and consider the partition of $[0,1)$ generated by $W_{n}(\theta)$ :

$$
\mathcal{P}_{n}(\theta)=\bigcup_{k=0}^{n} I_{k, n}, \quad \text { where } \quad I_{k, n}=\left[y_{k, n}, y_{k+1, n}\right) .
$$

It is a well known property of the Weyl sequence (see e.g. [3], [4]) that for any $n \geq 1$ the partition $\mathcal{P}_{n}(\theta)$ of $[0,1)$ contains the intervals $I_{k, n}$ whose lengths $\left|I_{k, n}\right|$ can only get two or three different values, namely, $\delta_{n}(\theta), \Delta_{n}(\theta)$ and perhaps $\delta_{n}(\theta)+\Delta_{n}(\theta)$.

1991 Mathematics Subject Classification: Primary 11K55. 
Set

$$
\alpha_{n}(\theta)=\min _{k=1, \ldots, n}\left|I_{k, n}\right|=\min \left\{\delta_{n}(\theta), \Delta_{n}(\theta)\right\}, \quad A_{n}(\theta)=\max _{k=1, \ldots, n}\left|I_{k, n}\right|,
$$

$\beta_{n}(\theta)=\max \left\{\delta_{n}(\theta), \Delta_{n}(\theta)\right\}, \quad \gamma_{n}(\theta)=\delta_{n}(\theta)+\Delta_{n}(\theta), \quad \xi_{n}(\theta)=\alpha_{n}(\theta) / \beta_{n}(\theta)$.

All these quantities, namely $\delta_{n}(\theta), \Delta_{n}(\theta), \alpha_{n}(\theta), A_{n}(\theta), \beta_{n}(\theta), \gamma_{n}(\theta)$ and $\xi_{n}(\theta)$, give a rather complete description of the partition $\mathcal{P}_{n}(\theta)$. We are interested in their asymptotic behaviour as $n \rightarrow \infty$. The main result of the paper is formulated in Theorem 1 below and presents the joint asymptotic distribution for $\left(n \delta_{n}(\theta), n \Delta_{n}(\theta)\right)$. In Corollaries 1-4 and Theorem 2 we derive the one-dimensional asymptotic distributions for all characteristics introduced above.

As demonstrated in Section 2, there is a close relationship between the Weyl and Farey sequences, and the quantities introduced above also characterize certain properties of the Farey sequences. (For example, $\alpha_{n}(\theta)$, whose asymptotic distribution has been derived in [2], characterizes the error in approximation of $\theta$ by the Farey sequence of order $n$ (see (11)).) The present paper thus also studies some distributional properties of the Farey sequences.

In what follows "meas" stands for the Lebesgue measure on $[0,1),\{\cdot\}$ and $\lfloor\cdot\rfloor$ denote the fractional and integer part operations respectively, $\varphi(\cdot)$ is the Euler totient function and $\operatorname{dilog}(\cdot)$ is the dilogarithm function:

$$
\operatorname{dilog}(t)=\int_{1}^{t} \frac{\log s}{1-s} d s .
$$

Also, we shall say that a sequence of functions $\psi_{n}(\theta), \theta \in[0,1)$, converges in distribution as $n \rightarrow \infty$ to a probability measure with a density $q(\cdot)$ if for any $t>0$,

$$
\lim _{n \rightarrow \infty} \operatorname{meas}\left\{\theta \in[0,1): \psi_{n}(\theta) \leq t\right\}=\int_{0}^{t} q(s) d s .
$$

The rest of the paper is organized as follows: the main results are formulated in Subsection 1.2, a relationship between the Weyl and Farey sequences is discussed in Section 2, all proofs are given in Section 3.

1.2. Formulation of the main results. For $0 \leq s, t<\infty$ define

$$
\Phi_{n}(s, t)=\operatorname{meas}\left\{\theta \in[0,1): n \delta_{n}(\theta) \leq s, n \Delta_{n}(\theta) \leq t\right\} .
$$

One can interpret $\Phi_{n}(\cdot, \cdot)$ as the two-dimensional cumulative distribution function (c.d.f.) of the random variables $n \delta_{n}(\theta)$ and $n \Delta_{n}(\theta)$, assuming that $\theta$ is uniformly distributed on $[0,1)$.

THEOREM 1. The sequence of functions $\Phi_{n}(\cdot, \cdot)$ pointwise converges, as $n \rightarrow \infty$, to the c.d.f. $\Phi(\cdot, \cdot)$ with density 
(2)

$$
\begin{aligned}
\phi(s, t) & =\frac{d^{2} \Phi(s, t)}{d s d t} \\
& =\frac{6}{\pi^{2} s t} \begin{cases}s+t-1 & \text { for } 0 \leq s, t \leq 1, s+t \geq 1, \\
s(1-s) /(t-s) & \text { for } 0 \leq s \leq 1 \leq t, \\
t(1-t) /(s-t) & \text { for } 0 \leq t \leq 1 \leq s, \\
0 & \text { otherwise. }\end{cases}
\end{aligned}
$$

This means that for all measurable sets $A$ in $\mathbb{R}^{2}$,

$$
\lim _{n \rightarrow \infty} \operatorname{meas}\left\{\theta \in[0,1):\left(n \delta_{n}(\theta), n \Delta_{n}(\theta)\right) \in A\right\}=\int_{A} \phi(s, t) d s d t .
$$

COROLlary 1. The sequences of functions $n \delta_{n}(\theta)$ and $n \Delta_{n}(\theta)$ converge in distribution, as $n \rightarrow \infty$, to the probability measure with density

$$
\phi_{\delta}(t)=\frac{6}{\pi^{2}} \begin{cases}0 & \text { for } t<0 \\ 1 & \text { for } 0 \leq t<1 \\ \frac{t-1}{t} \log \frac{t-1}{t}+\frac{1}{t} & \text { for } t \geq 1\end{cases}
$$

The proof of Corollary 1 consists in computation of $\int_{0}^{\infty} \phi(s, t) d s$ where $\phi(\cdot, \cdot)$ is defined in $(2)$.

COROLlary 2. The sequence of functions $n \alpha_{n}(\theta)$ converges in distribution, as $n \rightarrow \infty$, to the probability measure with density

$$
\phi_{\alpha}(t)=\frac{12}{\pi^{2}} \begin{cases}1 & \text { for } 0 \leq t<1 / 2 \\ \frac{1-t}{t}\left(1-\log \frac{1-t}{t}\right) & \text { for } 1 / 2 \leq t<1 \\ 0 & \text { otherwise }\end{cases}
$$

(Note again that Corollary 2 has been proved in [2], by different arguments.)

COROLlaRY 3 . The sequence of functions $n \beta_{n}(\theta)$ converges in distribution, as $n \rightarrow \infty$, to the probability measure with density

$$
\phi_{\beta}(t)=\frac{12}{\pi^{2}} \begin{cases}0 & \text { for } t<1 / 2, \\ \frac{1-t}{t} \log \frac{1-t}{t}-\frac{1}{t}+2 & \text { for } 1 / 2 \leq t<1, \\ \frac{t-1}{t} \log \frac{t-1}{t}+\frac{1}{t} & \text { for } t \geq 1 .\end{cases}
$$

COROLlary 4. The sequences of functions $n \gamma_{n}(\theta)$ and $n A_{n}(\theta)$ converge in distribution, as $n \rightarrow \infty$, to the probability measure with density

(6) $\phi_{\gamma}(t)=\frac{12}{\pi^{2}} \begin{cases}0 & \text { for } t<1, \\ \frac{t-2}{2 t} \log |t-2|-\frac{t-1}{t} \log (t-1)+\frac{1}{2} \log t & \text { for } t \geq 1 .\end{cases}$

To make the difference between the asymptotic behaviour of $\delta_{n}, \beta_{n}$ and $\gamma_{n}$ transparent, we provide Figure 1 which depicts the densities $\phi_{\delta}$, $\phi_{\beta}$ and $\phi_{\gamma}$. 


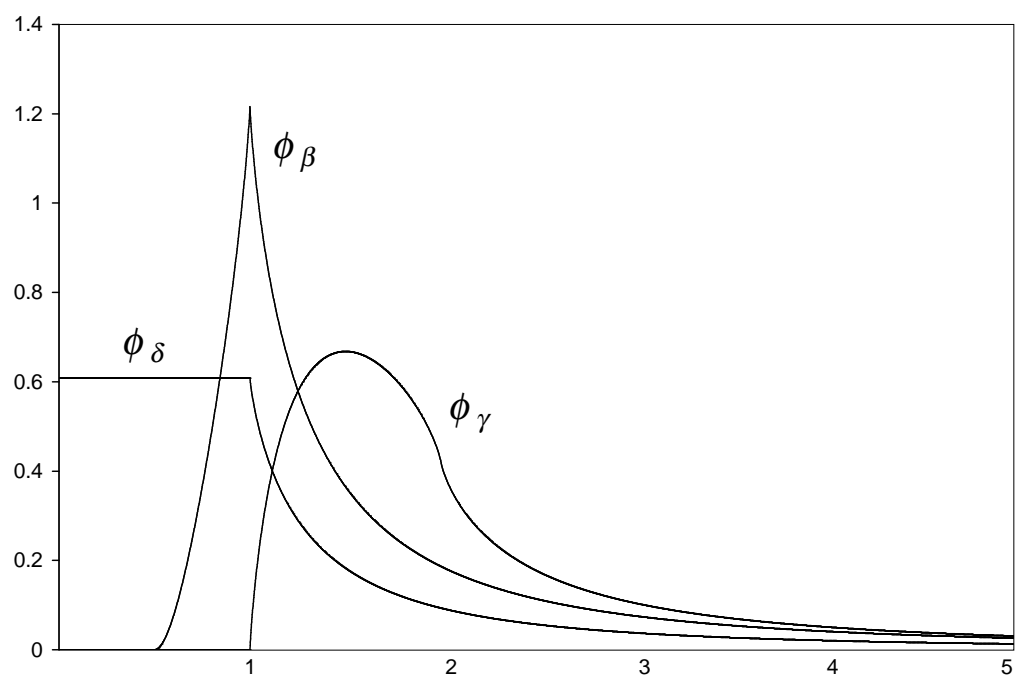

Fig. 1. Asymptotic densities for $n \delta_{n}(\theta), n \beta_{n}(\theta)$ and $n \gamma_{n}(\theta)$

THEOREM 2. The sequence of functions $\xi_{n}(\theta)$ converges in distribution, as $n \rightarrow \infty$, to the probability measure with density

$$
\phi_{\xi}(t)=-\frac{12}{\pi^{2}}\left(\frac{\log t}{1-t}+\frac{\log (1+t)}{t}\right), \quad t \in[0,1) .
$$

Theorem 2 can certainly be deduced from Theorem 1 . This however would require evaluation of an unpleasant integral; in Section 3 we instead give a straightforward proof.

\section{Relationships with the Farey sequences and continued fractions}

2.1. Relationship with the Farey sequences. The Farey sequence of order $n$, denoted by $\mathcal{F}_{n}$, is the collection of all rationals $p / q$ with $p \leq q \operatorname{gcd}(p, q)$ $=1$ and $1 \leq q \leq n$. The numbers in $\mathcal{F}_{n}$ are arranged in increasing order, and 0 and 1 are included in $\mathcal{F}_{n}$ as $0 / 1$ and $1 / 1$ respectively. There are $\left|\mathcal{F}_{n}\right|=N(n)+1$ points in $\mathcal{F}_{n}$ where

$$
N(n)=\sum_{q=1}^{n} \varphi(q)=\frac{3}{\pi^{2}} n^{2}+O(n \log n), \quad n \rightarrow \infty .
$$

The following well known statement establishes an important relationship between the Weyl and Farey sequences.

LEMma 1 (e.g. [3]). Let $\theta$ be an irrational number in $[0,1)$ and $W_{n}(\theta)$ be the Weyl sequence of order $n$. Let $\{q \theta\}$ and $\left\{q^{\prime} \theta\right\}$ correspond respectively to 
the smallest and largest members of $W_{n}(\theta)$ :

$$
y_{1}=\delta_{n}(\theta)=\{q \theta\}, \quad y_{n}=1-\Delta_{n}(\theta)=\left\{q^{\prime} \theta\right\} .
$$

Define $p=\lfloor q \theta\rfloor$ and $p^{\prime}=1+\left\lfloor q^{\prime} \theta\right\rfloor$. Then $p / q$ and $p^{\prime} / q^{\prime}$ are the consecutive fractions in the Farey sequence $\mathcal{F}_{n}$ such that $p / q<\theta<p^{\prime} / q^{\prime}$.

Let us rewrite the quantities (1) in terms of the Farey fractions $p / q$ and $p^{\prime} / q^{\prime}$ introduced in Lemma 1:

$$
\begin{aligned}
\delta_{n}(\theta) & =\{q \theta\}=q \theta-\lfloor q \theta\rfloor=q \theta-p, \\
\Delta_{n}(\theta) & =1-\left\{q^{\prime} \theta\right\}=1+\left\lfloor q^{\prime} \theta\right\rfloor-q^{\prime} \theta=p^{\prime}-q^{\prime} \theta .
\end{aligned}
$$

This in particular implies

$$
\alpha_{n}(\theta)=\min _{p / q \in \mathcal{F}_{n}}|q \theta-p| .
$$

2.2. An asymptotic property of the Farey sequences. In the sequel we shall use an asymptotic property of the Farey sequences formulated as Lemma 2.

If $p / q$ and $p^{\prime} / q^{\prime}$ are two consecutive Farey fractions in $\mathcal{F}_{n}$ then we call $\left(q, q^{\prime}\right)$ a neighbouring pair of denominators. It is easy to verify that for a fixed $n$ the set of all neighbouring pairs of denominators is

$$
Q_{n}=\left\{\left(q, q^{\prime}\right): q, q^{\prime} \in\{1, \ldots, n\}, \operatorname{gcd}\left(q, q^{\prime}\right)=1, q+q^{\prime}>n\right\},
$$

and these pairs, properly normalised, share the asymptotic two-dimensional uniformity. Specifically, the following result holds.

LEMma 2 (see [1]). Let $\nu_{n}$ be the two-variate probability measure assigning equal masses $1 / N(n)$ to the pairs $\left(q / n, q^{\prime} / n\right)$, where $\left(q, q^{\prime}\right)$ take all possible values in $Q_{n}$. Then the sequence of probability measures $\left\{\nu_{n}\right\}$ weakly converges, as $n \rightarrow \infty$, to the uniform probability measure on the triangle $T=\{(x, y): 0 \leq x, y \leq 1, x+y \geq 1\}$, that is, for any continuous function $f$ on $\mathbb{R}^{2}$,

$$
\frac{1}{N(n)} \sum_{\left(q, q^{\prime}\right) \in Q_{n}} f\left(q / n, q^{\prime} / n\right) \rightarrow 2 \iint_{T} f(x, y) d x d y, \quad n \rightarrow \infty .
$$

2.3. Association with continued fractions. Let us now indicate an interesting analogy between the quantity $\xi_{n}(\theta)$ and the residuals in the continued fraction expansions.

Let $\theta$ be an irrational number in $[0,1)$. We denote by $\theta=\left[a_{1}, a_{2}, \ldots\right]$ its continued fraction expansion and by $p_{n} / q_{n}=\left[a_{1}, a_{2}, \ldots, a_{n}\right]$ its $n$th convergent.

Let also

$$
r_{0}=\theta, \quad r_{n}=\left\{1 / r_{n-1}\right\} \quad \text { for } n=1,2, \ldots
$$

be the associated dynamical system. 
As is well known, the asymptotic density of $\left\{r_{n}\right\}$ is

$$
p(t)=\frac{1}{\log 2} \cdot \frac{1}{1+t}, \quad 0 \leq t<1 .
$$

For every $n \geq 0, r_{n}=r_{n}(\theta)$ allows the following continued fraction expansion: $r_{n}(\theta)=\left[a_{n+1}, a_{n+2}, \ldots\right]$. It is not difficult to check (see e.g. [4]), that

$$
r_{n}(\theta)=\frac{\left|q_{n} \theta-p_{n}\right|}{\left|q_{n-1} \theta-p_{n-1}\right|}, \quad n>1 .
$$

The role of $r_{n}(\theta)$ for $\mathcal{F}_{n}$ is played by

$$
\xi_{n}(\theta)=\frac{\min \left(|q \theta-p|,\left|q^{\prime} \theta-p^{\prime}\right|\right)}{\max \left(|q \theta-p|,\left|q^{\prime} \theta-p^{\prime}\right|\right)}=\frac{\alpha_{n}(\theta)}{\beta_{n}(\theta)},
$$

where $p / q, p^{\prime} / q^{\prime}$ are the members of $\mathcal{F}_{n}$ neighbouring to $\theta$. Figure 2 compares the asymptotic densities for $r_{n}(\theta)$ and $\xi_{n}(\theta)$.



Fig. 2. Asymptotic densities for $r_{n}(\theta)$ and $\xi_{n}(\theta)$

\section{Proofs}

3.1. Proof of Theorem 1. Consider the two-variate function

$$
\widetilde{\Phi}_{n}(s, t)=\operatorname{meas}\left\{\theta \in[0,1): n \delta_{n}(\theta)>s, n \Delta_{n}(\theta)>t\right\},
$$

where $0 \leq s, t<\infty$. The c.d.f. $\Phi(s, t)$ is related to $\widetilde{\Phi}(s, t)$ through the inclusion-exclusion formula

$$
\Phi(s, t)=1-\widetilde{\Phi}(s, 0)-\widetilde{\Phi}(0, t)+\widetilde{\Phi}(s, t) .
$$

Let $p / q$ and $p^{\prime} / q^{\prime}$ be consecutive fractions in $\mathcal{F}_{n}$. Define points $\theta_{1}, \theta_{2}$ in $\left[p / q, p^{\prime} / q^{\prime}\right]$ such that

$$
n \delta_{n}\left(\theta_{1}\right)=s, \quad n \Delta_{n}\left(\theta_{2}\right)=t
$$


It is easily seen that

$$
\begin{aligned}
\operatorname{meas}\left\{\theta \in\left[p / q, p^{\prime} / q^{\prime}\right]: n \delta_{n}(\theta)>s, n \Delta_{n}(\theta)>t\right\} & \\
& = \begin{cases}\theta_{2}-\theta_{1} & \text { for } \theta_{2}-\theta_{1}>0, \\
0 & \text { for } \theta_{2}-\theta_{1} \leq 0 .\end{cases}
\end{aligned}
$$

We now try to find a simple expression for the difference $\theta_{2}-\theta_{1}$. First, formulas (9) and (10) yield

$$
\theta_{1}=\frac{s / n+p}{q}, \quad \theta_{2}=\frac{p^{\prime}-t / n}{q^{\prime}},
$$

and therefore

$$
\theta_{2}-\theta_{1}=\frac{p^{\prime}-t / n}{q^{\prime}}-\frac{s / n+p}{q}=\frac{1}{q q^{\prime}}\left(1-\frac{t q}{n}-\frac{s q^{\prime}}{n}\right) .
$$

We thus get

$$
\widetilde{\Phi}_{n}(s, t)=\sum_{\left(q, q^{\prime}\right) \in Q(n, s, t)} \frac{1}{q q^{\prime}}\left(1-\frac{t q}{n}-\frac{s q^{\prime}}{n}\right)
$$

where

$$
Q(n, s, t)=\left\{\left(q, q^{\prime}\right) \in Q_{n}: 1-t q / n-s q^{\prime} / n>0\right\} .
$$

Using formula (8) we have

$\widetilde{\Phi}_{n}(s, t)=\frac{3}{\pi^{2} N(n)} \sum_{\left(q, q^{\prime}\right) \in Q(n, s, t)} \frac{n^{2}}{q q^{\prime}}\left(1-\frac{t q}{n}-\frac{s q^{\prime}}{n}\right)+O\left(n^{-1} \log n\right), \quad n \rightarrow \infty$.

Applying Lemma 2 we get

$$
\widetilde{\Phi}_{n}(s, t) \rightarrow \widetilde{\Phi}(s, t)=\frac{6}{\pi^{2}} \iint_{Q(s, t)}\left(\frac{1-t x-s y}{x y}\right) d x d y
$$

where

$$
Q(s, t)=\{x, y: 0 \leq x, y \leq 1, x+y \geq 1,1-t x-s y>0\} .
$$

The formula for the integral on the right-hand side of (13) can be rewritten differently in 5 different regions:

1. For $s+t \leq 1$ :

$$
\widetilde{\Phi}(s, t)=\frac{6}{\pi^{2}} \int_{0}^{1} \int_{-y}^{1}\left(\frac{1-t x-s y}{x y}\right) d x d y=1-\frac{6}{\pi^{2}}(s+t) .
$$


2. For $0 \leq s, t \leq 1, s+t>1$ :

$$
\begin{aligned}
\widetilde{\Phi}(s, t)= & \frac{6}{\pi^{2}} \int_{0}^{(1-s) / t} \int_{1-y}^{1}\left(\frac{1-t x-s y}{x y}\right) d x d y \\
& +\frac{6}{\pi^{2}} \int_{(1-s) / t}^{1} \int_{1-y}^{(1-y t) / s}\left(\frac{1-t x-s y}{x y}\right) d x d y \\
= & -\frac{12}{\pi^{2}}+\frac{6}{\pi^{2}}\left(s+t+(1+\log s-s) \log \frac{1-s}{t}\right. \\
& \left.+(1+\log t-t) \log \frac{1-t}{s}+\log s \log t+\operatorname{dil} \log s+\operatorname{dil} \log t\right) .
\end{aligned}
$$

3. For $s>1, t \leq 1$ :

$$
\begin{aligned}
\widetilde{\Phi}(s, t)= & \frac{6}{\pi^{2}} \int_{(s-1) /(s-t)}^{1} \int_{1-y}^{(1-y t) / s}\left(\frac{1-t x-s y}{x y}\right) d x d y \\
= & 1-\frac{6}{\pi^{2}}+\frac{6}{\pi^{2}}\left(t+(s-\log s-t) \log \frac{s-t}{s-1}+(1-t) \log \frac{s-1}{s}\right. \\
& \left.-\operatorname{dil} \log (1-t)+\operatorname{dil} \log \frac{s(1-t)}{s-t}-\operatorname{dilog} \frac{1-t}{s-t}\right) .
\end{aligned}
$$

4. For $s \leq 1, t>1$ : Analogously to the previous case with the replacement $s \leftrightarrow t$.

5. For $s>1, t>1: \widetilde{\Phi}(s, t)=0$.

Using formula (12) we can find the density

$$
\phi(s, t)=\frac{d \Phi(s, t)}{d s d t}=\frac{d \widetilde{\Phi}(s, t)}{d s d t}
$$

of the joint asymptotic distribution. Calculation then gives (2).

3.2. Proof of Corollary 2. The function $\alpha_{n}(\theta)=\min \left\{\delta_{n}(\theta), \Delta_{n}(\theta)\right\}$ is measurable with respect to $\mathcal{B}$, the $\sigma$-algebra of Borel subsets of $[0,1)$, and it can be associated with the probability measure $d \Phi_{n}^{\alpha}(t), 0 \leq t<\infty$, where

$$
\begin{aligned}
\Phi_{n}^{\alpha}(t) & =\operatorname{meas}\left\{\theta \in[0,1): n \alpha_{n}(\theta) \leq t\right\} \\
& =1-\operatorname{meas}\left\{\theta \in[0,1): n \min \left(\delta_{n}(\theta), \Delta_{n}(\theta)\right)>t\right\} \\
& =1-\operatorname{meas}\left\{\theta \in[0,1): n \delta_{n}(\theta)>t, n \Delta_{n}(\theta)>t\right\} .
\end{aligned}
$$

Therefore, for all $0 \leq t<\infty$,

$$
\Phi_{n}^{\alpha}(t) \rightarrow \Phi^{\alpha}(t)=1-\widetilde{\Phi}(t, t), \quad n \rightarrow \infty .
$$


Calculation gives

$$
\Phi^{\alpha}(t)= \begin{cases}\frac{12}{\pi^{2}} t & \text { for } 0 \leq t<1 / 2, \\ \frac{12}{\pi^{2}}\left(-t+\log \frac{1-t}{t}(t-\log t-1)+\operatorname{dilog} \frac{1}{t}\right)+\frac{12}{\pi^{2}}+1 \\ 1 & \text { for } 1 / 2 \leq t<1, \\ 1 & \text { for } t \geq 1 .\end{cases}
$$

Differentiation gives the expression (4) for the density $\phi_{\alpha}(t)=d \Phi^{\alpha}(t) / d t$.

3.3. Proof of Corollary 3. The function $\beta_{n}(\theta)=\max \left\{\delta_{n}(\theta), \Delta_{n}(\theta)\right\}$ is $\mathcal{B}$-measurable. We then have, for all $0 \leq t<\infty$,

$$
\begin{aligned}
\Phi_{n}^{\beta}(t) & =\operatorname{meas}\left\{\theta \in[0,1): n \beta_{n}(\theta) \leq t\right\} \\
& =\operatorname{meas}\left\{\theta \in[0,1): n \delta_{n}(\theta) \leq t, n \Delta_{n}(\theta) \leq t\right\} .
\end{aligned}
$$

Therefore, for all $0 \leq t<\infty$,

$$
\Phi_{n}^{\beta}(t) \rightarrow \Phi^{\beta}(t)=\Phi(t, t), \quad n \rightarrow \infty .
$$

Calculation gives

$$
\Phi^{\beta}(t)=\left\{\begin{array}{lc}
0 & \text { for } 0 \leq t<1 / 2, \\
\frac{12}{\pi^{2}}\left(2 t-\log \frac{1-t}{t}(t-\log t-1)-\operatorname{dilog} \frac{1}{t}\right)-\frac{12}{\pi^{2}}-1 & \text { for } 1 / 2 \leq t<1, \\
\frac{12}{\pi^{2}}\left(\log \frac{t-1}{t}(t-\log t-1)+\operatorname{dilog} \frac{1}{t}\right)+\frac{12}{\pi^{2}}-1 & \text { for } t \geq 1 .
\end{array}\right.
$$

Differentiation gives the expression (5) for the density $\phi_{\beta}(t)=d \Phi^{\beta}(t) / d t$.

3.4. Proof of Corollary 4. Analogously to the proofs of Corollaries 2 and 3 , the sequence of c.d.f.

$$
\Phi_{n}^{\gamma}(t)=\operatorname{meas}\left\{\theta \in[0,1): n \gamma_{n}(\theta) \leq t\right\}, \quad 0 \leq t<\infty,
$$

pointwise converges to the c.d.f.

$$
\Phi^{\gamma}(t)=\iint_{S(t)} \phi(x, y) d x d y, \quad 0 \leq t<\infty
$$

where

$$
S(t)=\{(x, y): 0 \leq x, y \leq 1,1 \leq x+y \leq t\} .
$$

Calculation yields (6).

The convergence of the sequence $n A_{n}(\theta)$ to the asymptotic distribution with density $\phi_{\gamma}$ follows from the just proved convergence of the sequence $n \gamma_{n}(\theta)$ to the same distribution and the fact that $A_{n}(\theta)=\gamma_{n+1}(\theta)$ for all $\theta \in(0,1)$ and all $n \geq n(\theta)=\max \{1 / \theta, 1 /(1-\theta)\}$. 
3.5. Proof of Theorem 2. The function $\xi_{n}(\theta)$ is $\mathcal{B}$-measurable. Define

$$
\Phi_{n}^{\xi}(t)=\operatorname{meas}\left\{\theta \in[0,1): \xi_{n}(\theta) \leq t\right\}, \quad 0 \leq t \leq 1 .
$$

Let $p / q, p^{\prime} / q^{\prime}$ be consecutive fractions in $\mathcal{F}_{n}$. Consider the behaviour of $\xi_{n}(\theta)$ in the interval $\left[p / q, p^{\prime} / q^{\prime}\right]$. Define the mediant $m=\left(p+p^{\prime}\right) /\left(q+q^{\prime}\right)$. Then for $\theta$ in $[p / q, m)$ we have $\delta_{n}(\theta)<\Delta_{n}(\theta)$, and for $\theta$ in $(m, p / q]$ we have $\delta_{n}(\theta)>\Delta_{n}(\theta)$ and $\delta_{n}(m)=\Delta_{n}(m)$, that is, $\xi_{n}(m)=1$.

If $t \in[0,1]$ is fixed then there is a unique point $\theta_{t}$ in $[p / q, m]$ such that

$$
\xi_{n}\left(\theta_{t}\right)=\frac{\delta_{n}\left(\theta_{t}\right)}{\Delta_{n}\left(\theta_{t}\right)}=t
$$

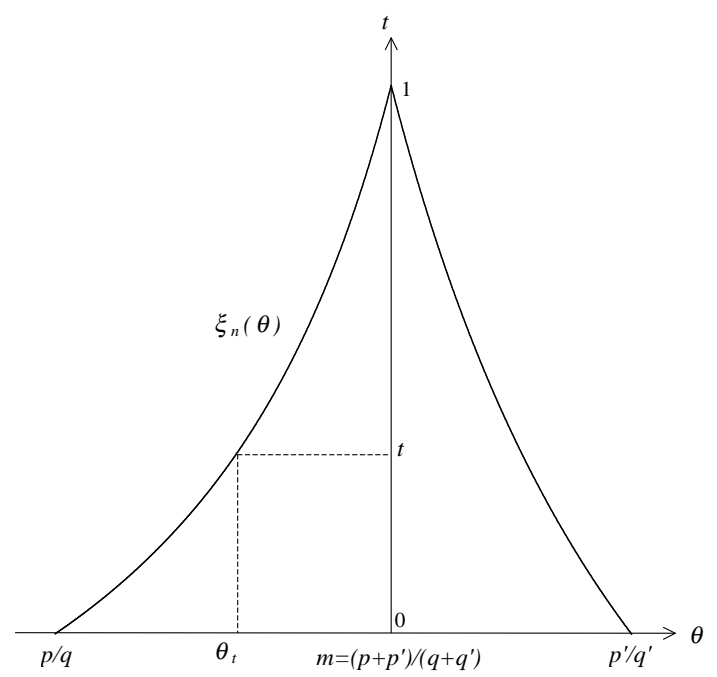

Fig. 3. Behaviour of the function $\xi_{n}(\theta)$ in the interval $\left[p / q, p^{\prime} / q^{\prime}\right]$

An easy observation shows (see Fig. 3) that

$$
\operatorname{meas}\left\{\theta \in[p / q, m]: \xi_{n}(\theta) \leq t\right\}=\theta_{t}-p / q .
$$

Formula (14) implies

$$
q \theta_{t}-p=t\left(p^{\prime}-q^{\prime} \theta_{t}\right), \quad \theta_{t}=\frac{p+t p^{\prime}}{q+t q^{\prime}} .
$$

Therefore,

$$
\operatorname{meas}\left\{\theta \in[p / q, m]: \xi_{n}(\theta) \leq t\right\}=\frac{p+t p^{\prime}}{q+t q^{\prime}}-\frac{p}{q}=\frac{t}{q\left(q+t q^{\prime}\right)} .
$$

We then get, for all $0 \leq t \leq 1$, 


$$
\begin{aligned}
\operatorname{meas}\left\{\theta \in[0,1): \xi_{n}(\theta)\right. & \leq t\} \\
& =2 \sum_{\left(q, q^{\prime}\right) \in Q_{n}} \frac{t}{q\left(q+t q^{\prime}\right)}=2 \sum_{\left(q, q^{\prime}\right) \in Q_{n}} \int_{0}^{t} \frac{d \tau}{\left(q+\tau q^{\prime}\right)^{2}} \\
& =2 \int_{0}^{t}\left(\sum_{\left(q, q^{\prime}\right) \in Q_{n}} \frac{1}{\left(q+\tau q^{\prime}\right)^{2}}\right) d \tau,
\end{aligned}
$$

where the factor 2 is due to the cases when $\delta_{n}(\theta)>\Delta_{n}(\theta)$.

Therefore, we can write, for all $0 \leq t \leq 1$,

$$
\Phi_{n}^{\xi}(t)=\int_{0}^{t} \phi_{n}^{\xi}(\tau) d \tau, \quad \text { where } \quad \phi_{n}^{\xi}(\tau)=2 \sum_{\left(q, q^{\prime}\right) \in Q_{n}} \frac{1}{\left(q+\tau q^{\prime}\right)^{2}} .
$$

Using formula (8) write

$$
\phi_{n}^{\xi}(\tau)=\frac{6}{\pi^{2} N(n)} \sum_{\left(q, q^{\prime}\right) \in Q_{n}} \frac{n^{2}}{\left(q+\tau q^{\prime}\right)^{2}}+O\left(n^{-1} \log n\right), \quad n \rightarrow \infty .
$$

Applying Lemma 2 we get

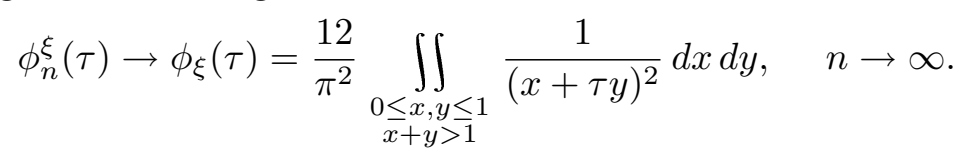

Calculation of the integral gives the expression (7) for the density.

\section{References}

[1] P. P. Kargaev and A. A. Zhigljavsky, Asymptotic distribution of the distance function to the Farey points, J. Number Theory 65 (1997), 130-149.

[2] H. Kesten, Some probabilistic theorems on Diophantine approximations, Trans. Amer. Math. Soc. 103 (1962), 189-217.

[3] N. B. Slater, Gaps and steps for the sequence $n \theta \bmod 1$, Proc. Cambridge Philos. Soc. 63 (1967), 1115-1123.

[4] V. T. Sós, On the distribution mod 1 of the sequence no, Ann. Univ. Sci. Budapest. Eötvös Sect. Math. 1 (1958), 127-134.

School of Mathematics

Cardiff University

Senghennydd Road

Cardiff CF2 4YH, U.K.

E-mail: ZhigljavskyAA@cardiff.ac.uk
Institute of Mathematics Polish Academy of Sciences P.O. Box 137 00-950 Warszawa, Poland E-mail: iskander@impan.gov.pl 